\title{
Use of requirements engineering within the Thirty Meter Telescope project
}

\author{
John Rogers ${ }^{\mathrm{a}}$, Hugh Thompson ${ }^{\mathrm{a}}$ \\ ${ }^{a}$ Thirty Meter Telescope Project, 2632 E. Washington Boulevard, Pasadena, CA, USA 91107
}

\begin{abstract}
The Thirty Meter Telescope is comprised of thirty five individual sub-systems which include optical systems, instruments, adaptive optics systems, controls, mechanical systems, supporting software and hardware and the infrastructure required to support their operation. These thirty five sub-systems must operate together as a system to enable the telescope to meet the science cases for which it is being developed. These science cases are formalized and expressed as science requirements by the project's Science Advisory Committee. From these, a top down requirements engineering approach is used within the project to derive consistent operational, architectural and ultimately detailed design requirements for the sub-systems. The various layers of requirements are stored within a DOORS requirements database that also records the links between requirements, requirement rationale and requirement history. This paper describes the development of the design requirements from science cases, the reasons for recording the links between requirements and the benefits that documenting this traceability will yield during the design and verification of the telescope. Examples are given of particular science cases, the resulting operational and engineering requirements on the telescope system and how individual sub-systems will contribute to these being met.
\end{abstract}

Keywords: TMT, system engineering, requirements

\section{INTRODUCTION}

The technical development of the Thirty Meter Telescope Observatory is being managed by employing a rigorous system engineering approach. System engineering encompasses many different activities, but success depends on a stable and complete set of requirements that are maintained throughout the project lifecycle. Recognizing this, a requirements engineering process has been employed within the TMT project since the conceptual design phase $\mathrm{e}^{[1],[2]}$. This activity began with the gathering of customer requirements and will only be complete once the as built observatory has demonstrated its ability to fulfill these requirements. Even beyond this, it can be anticipated that the requirements that have been used to design the observatory will act as a source of reference for those operating and using the observatory. Given their importance and longevity, we are devoting much care and effort throughout the project to developing and maintaining the correct requirements. To assist in this effort and to ensure that all the information supporting the requirements is captured, a plan has been developed to explain how requirements will be managed, how the traceability will be developed and recorded and how the information can be used to benefit the project. This paper summarizes the progress made so far in developing the overall TMT requirement set and the plans to use the requirements and associated traceability information.

\section{TMT REQUIREMENTS HIERARCHY}

The requirements for the Thirty Meter Telescope are contained in requirement documents organized into four distinct levels as shown in figure 1. It is important that the content and scope of each of these levels is defined in advance of writing the documents as it affects the level of detail that must be contained within each requirement document, the timeline for developing and releasing the documents and the level of requirement change or evolution that can be expected. 


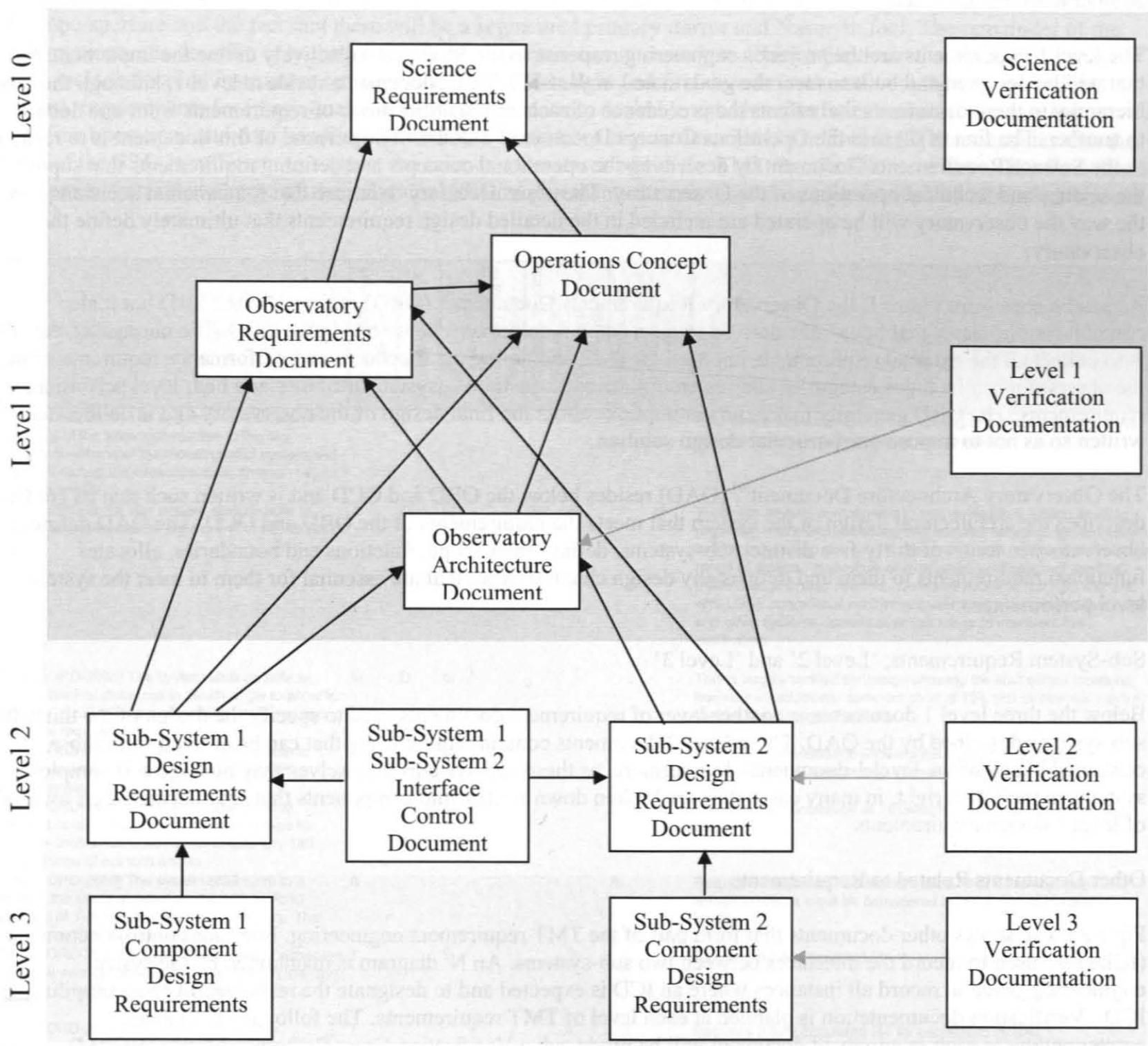

Figure 1 - TMT Requirement Document Hierarchy, arrows indicated DOORS links

Science Requirements, 'Level 0’

At the highest level of the TMT requirements are the science requirements which can be considered as analogous to the customer or user requirements of a typical system. The TMT science requirements are contained in the Science Requirements Document (SRD) ${ }^{[3]}$. This document is owned and developed by the Project Scientist and Science Advisory Committee and contains requirements that define observatory capabilities that directly support the envisaged TMT science cases defined in the TMT Detailed Science $\operatorname{Case}^{[8]}$. It should be emphasized that the science cases described have been carefully defined so that the observatory will be built to allow exploration rather than support specific experiments. The intention is to ensure that TMT's capabilities will be as wide as possible so as to support the inevitable evolution of science cases over its lifetime. 


\section{System Requirements, 'Level 1'}

The level 1 requirements are the project's engineering response to the SRD and collectively define the implementation that will be designed and built to meet the goals stated in the SRD. Three documents reside at level 1, although there is a hierarchy to these documents that affects the precedence of each and the flow down of requirements from one document to another. The first of these is the Operations Concept Document ${ }^{[4]}(\mathrm{OCD})$. The purpose of this document is to respond to the Science Requirements Document by describing the operational concepts and defining requirements that support the science and technical operations of the Observatory. These are necessary to ensure that fundamental decisions about the way the observatory will be operated are included in the detailed design requirements that ultimately define the observatory.

As can be seen from figure 1, the Observatory Requirements Document ${ }^{[5]}(\mathrm{ORD})$ responds to the SRD but it also contains requirements that have been derived to meet some requirements contained in the OCD. The purpose of the ORD is to collect all the external requirements imposed on TMT and define the functional and performance requirements of the observatory; it includes categories such as environmental constraints, system attributes and high level performance requirements. The ORD generally makes no assumptions about the final design of the observatory and is deliberately written so as not to impose one particular design solution.

The Observatory Architecture Document ${ }^{[6]}(\mathrm{OAD})$ resides below the ORD and OCD and is written such that its content describes the architectural design of the system that meets the requirements of the ORD and OCD. The OAD defines the observatory in terms of thirty five distinct sub-systems, defines their scope, functions and boundaries, allocates functional requirements to them and defines any design characteristics that are essential for them to meet the system level performance.

\section{Sub-System Requirements, 'Level 2' and 'Level 3'}

Below the three level 1 documents is another layer of requirement documents used to specify the design of the thirty five sub-systems described by the OAD. These level 2 documents contain requirements that can be derived from those contained in any of the level 1 documents. Furthermore, as these sub-systems themselves may be large and complex systems in their own right, in many cases they are broken down further into components that are allocated their own set of level 3 design requirements.

\section{Other Documents Related to Requirements}

Figure 1 also shows other documents that form part of the TMT requirement engineering. Interface control documents (ICDs) are used to record the interfaces between two sub-systems. An $\mathrm{N}^{2}$ diagram is maintained by the system engineering group to record all instances where an ICD is expected and to designate the responsibility for compiling the ICD. Verification documentation is planned at each level of TMT requirements. The following documents corresponding to each requirement document will be produced; a Verification Cross Reference Matrix (VCRM) summarizing the verification method planned by major milestones (verification event) during the design and development of the sub-system or system; Verification Plans that contain a high level summary of the verification planned at each verification event together with the rationale for selecting the particular method at this event; Verification Procedures and Reports that detail the procedure for performing the verification and record the results, thereby forming the verification report. An extract from the proposed VCRM supporting ORD verification is included as figure 2 .

\section{DEVELOPMENT OF SUB-SYSTEM REQUIREMENTS FROM SCIENCE CASE}

As described in the Science Flowdown for the Thirty Meter Telescope ${ }^{[7]}$, the process of developing the SRD began in about 2000 by writing a set of initial science cases. The first version of the SRD contained descriptions of and requirements for a set of instruments and systems that could support these science cases. These were used as the basis for instrument feasibility studies in 2005 and 2006 which resulted in more detailed science cases and operational concepts and lead to an updated version of the SRD. 
The SRD is written assuming very little about the final design of the system other than key optical characteristics such as telescope aperture and the fact that there will be a segmented primary mirror and Nasmyth foci. The remainder of the SRD requirements describe only the performance characteristics necessary to achieve the desired science performance. These include detailed instrument requirements for the first light and first decade instrument configurations and $\mathrm{AO}$ systems, requirements for image quality and telescope motion, desirable site characteristics and requirements for nighttime operations and data handling. The science flow down matrix described in reference [7] demonstrates that the scope of the SRD is sufficient to support the science cases described in the TMT Detailed Science Case. The flow down also demonstrates that almost all the individual SRD requirements directly enable or support the TMT Detailed Science Case $^{[8]}$.

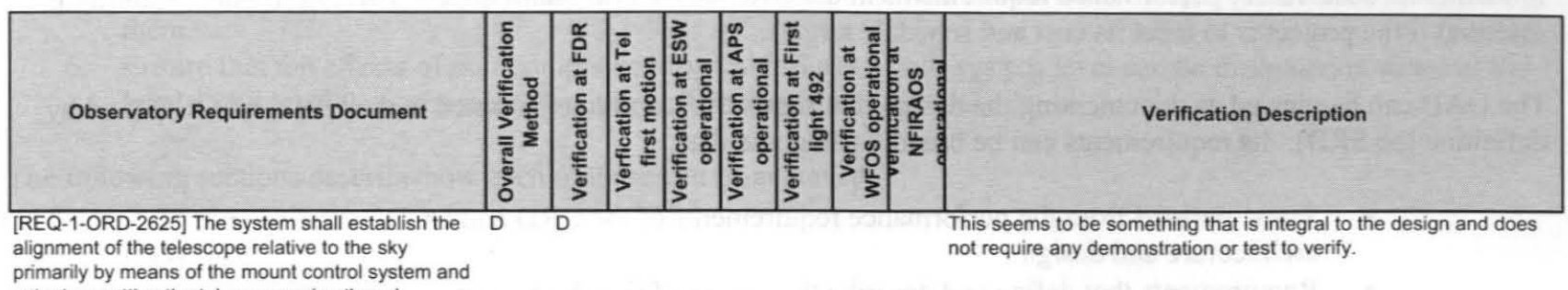

actuators setting the telescope azimuth and

elevation angles.

[REQ-1-ORD-2630] The system shall be able to

observe anywhere on the sky from $1^{\circ}$ to $65^{\circ}$ zenith

A D $\quad \mathrm{I}, \mathrm{M}$

M

M There are several components to this verification, design aspects regarding limit switch locations and actuator stroke range are shown at FDR. Demonstration at TEL first motion will confirm the designed range of motion. Inspection of subsystem verification of the ENC motion range is also needed. Demonstrations at first light 492 and with LGSF operational confirm actuator stroke budget and that LGSF and other systems operate over this range to complete the verification.

[REQ-1-ORD-2635] The system shall be able to M D M move additional distances in zenith angle to allow for servicing, maintenance and parking positions, and safety, as required.

[REQ-1-ORD-2640] The system shall be able to M M observe anywhere on the sky from $0^{\circ}$ to $360^{\circ}$ azimuth angle.

[REQ-1-ORD-2645] The system shall provide M M

This is largely verified by design showing the limit switch locations, however an additional demonstration at TEL first motion will confirm the designed range of motion.

This is by demonstration showing the azimuth wrap motion limitations at TEL first motion to confirm the design range.

sufficient continuous azimuth range coverage to

enable a continuous observation across any 180

degree range of azimuth angles.

[REQ-1-ORD-2650] The system shall point to a

target in the whole accessible sky with an RMS

accuracy of 1 arcsec in each axis on the sky. The

goal is to achieve 0.5 arcsec RMS.

[REQ-1-ORD-2652] A 5 arcminute move of either the T

telescope azimuth or elevation axes (not on the sky)

shall be completed within 1.5 seconds.

[REQ-1-ORD-2654] A $1^{\circ}$ move of either the T

telescope azimuth or elevation axes (not on the sky)

shall be completed within 5 seconds.

[REQ-1-ORD-2656] The long term average slew time A

between science targets shall be less than 60

seconds.

A
This is by demonstration at TEL first motion to confirm the design range.

Requires analysis as requirement is on RMS accuracy, also sampling issues must be considered in the roll-up of the tested targets.

T

Requires a fully populated M1 for verification by test.

Requires a fully populated M1 for verification by test.

Largely this is by design. A report will show that particular acceleration and maximum velocity values enable the requirement to be met. A confirmation test that those accelerations are achieved with a fully populated M1 is the final verification step, however this is expected to be a STR subsystem verification, so at system level this last step is an inspection of a lower level verification result.

Figure 2, Extract from proposed verification cross reference matrix supporting ORD verification

The next level of requirement flow down is from the SRD to the ORD and OCD. Similar to the SRD, the ORD and OCD assume very little about the design. Their role is to translate the SRD's science requirements to the 'real' engineering requirements. There is quite a direct and obvious link between the science requirements in the SRD and the ORD and OCD and in some cases where the SRD is sufficiently detailed they merely repeat what is stated in the SRD. In other cases the SRD requirements have been developed and converted into terms that can be used as the basis for developing system wide budgets. 
At this point, our process allows for differences to exist between the SRD and level 1 documents. These are assessed by the TMT Science Advisory Committee to confirm that the ORD requirement set will enable the observatory to achieve the stated science goals. The differences between the documents are tracked and regularly reviewed to ensure that the observatory design continues to offer the best solution to the science requirements whilst respecting program constraints. This process will lead to resolution of the differences between the documents.

As the OCD and ORD requirements don't describe the design of the observatory it is expected that significant changes to them will be infrequent and result only from planned developments in the project. For example the selection of Mauna Kea as the TMT site lead to significant changes to the environmental conditions. No significant changes to the fundamental observatory performance requirements in the ORD or OCD are anticipated. This requirement stability is essential if the project is to meet its cost and schedule targets.

The OAD can be viewed as documenting the design that meets the requirements stated in the ORD and OCD (and by definition the SRD). Its requirements can be broadly categorized as:

- Those derived from the performance requirements of the ORD and OCD but being specific to the TMT architecture and design

- Requirements that define and describe the design of the observatory

- Definition of high level interfaces between subsystems either as architectures (for example wavefront control, cooling, power distribution) or as specifications to ensure consistency across subsystems (e.g. space envelopes)

The first category includes items such as performance budgets where a top level ORD performance requirement is broken down and allocated to each sub-system that contributes to achieving that performance. The second category includes the definition of design parameters that are essential to meet the performance requirements (e.g. the optical prescription), whilst the third defines characteristics that potentially affect the ability of the system or other sub-systems to meet their requirements (e.g. crane capacities and locations) or where changes to the design would affect the design of other sub-systems (e.g. pupil obscuration pattern, control architecture etc.). The OAD will also eventually contain the 'Unknowable' requirements. These are requirements that aren't initially envisaged because they are specific to the design of the telescope, but whose definition is essential to the accomplishment of the higher level ORD and OCD requirements.

From these descriptions of OAD requirements it can be seen that they describe the final design of TMT. It can be expected therefore that the $\mathrm{OAD}$ requirements will continue to evolve as the Observatory converges on its final design. Rather than adding functionality (which would have cost or schedule effects), this process can be viewed as adding necessary definition or clarifying and improving existing requirements. The TMT Change Control process for changes to the ORD, OCD and OAD is well defined and exercised and ensures that changes are agreed to by the appropriate TMT project staff, are communicated to all members of the TMT project and are evaluated for potential effects to the project cost and schedule.

The sub-system design requirement documents (DRDs) that make up the level 2 documents include requirements that flow from any of the three level 1 documents. The sub-system component requirements that make up the level 3 documents must be traceable only to their parent level 2 requirement. The goal for these level 2 and level 3 requirements is that when they reach their lowest level (i.e. they have no 'children'), the requirements are unambiguous and approaching an 'atomic' or indivisible state.

\section{RECORDING THE PROJECT REQUIREMENTS IN DOORS}

\subsection{Goals for Documenting the Traceability}

As the requirements flow down from one requirement level to the next, both the number of requirements and the level of detail included in the requirements increases. By recording the links between levels of requirements as they are developed we will: 
1. Track how our technical requirements and their design solution meet the high level technical requirements and the project science goals and report this to the TMT science advisory committee.

2. Ensure the completeness of sub-system DRDs by allocating level 1 requirements to sub-systems and checking that they are covered appropriately at level 2

3. Confirm that the level 1 requirements, in particular the set of requirements in the OAD are complete and detailed enough to allow level 2 DRDs to be written

4. Develop a picture of how the verification of level 1 requirements is achieved incrementally through the verification of requirements at level 2 and level 3

5. Report the effects of changes to high level documents on the level 2 and level 3 requirements that flow from them

6. Ensure that the effects of non-compliances or test failures at sub-system level can be evaluated in terms of the level 1 and level 0 requirements that they contribute to

The following sections describe how each of these can be achieved.

\subsection{Overview}

The TMT project employs DOORS as a requirements management tool that allows the project requirements from level 0 to level 3 to be stored in a single database. We have found that the major benefits in using DOORS compared to simply using text requirements documents are:

- The ability to link requirements; requirements development can be seen as a cascade of a small number of high level 'customer' requirements (science requirements in the case of TMT) into successively lower levels of requirements. As the requirements flow into lower levels the requirements become more detailed, more specific to the system design and rapidly increase in number. DOORS allows links to be created between the different levels of requirement so that it is possible to find all the requirements that are derived from a single high level requirement.

- Recording of metadata; a requirements document generally only records the requirement text and in some cases additional discussion where this aids with the understanding or intent of the requirement. There is often more data associated with the requirement that may be impractical to include in the requirement document but can easily be stored in the DOORS database. The categories of metadata associated with each requirement are explained further in section 4.5 .

- Recording of verification and compliance information; at various milestones throughout the project the system and sub-systems are required to report compliance status, provide verification plans and report verification results. By assigning specific attributes to each requirement, these compliance and verification reports can be produced by exporting specific attributes from DOORS.

- Creating links between the requirements and other related design documents; for example a requirement may be dependant on the correct implementation of an interface to another sub-system. DOORS allows the requirement and interface to be linked so that this interface information can be easily viewed alongside the requirement and checked for consistency. Similarly verification reports will be included in DOORS and linked to requirements.

\subsection{Storing the Requirement Data}

The DOORS database is used to store the requirements of the project. These requirements are generally developed and documented outside the DOORS database, i.e. in Word documents that are stored and controlled via TMT's document control system and are available throughout the project. To avoid confusion in the case that requirements in the Word document are in conflict with those stored in the DOORS database, the Word documents are considered as the official reference source for the requirements. As requirement documents are updated, the affected requirements in the DOORS database are updated to maintain consistency. 


\subsection{Requirement Links}

As explained in section 2, there is a well understood hierarchy to the TMT documentation and the flow down of requirements and hence links between requirements are expected to be consistent with this. For example a requirement in a level 3 document could not be linked directly to a requirement in a level 1 document, the link must be established via the level 2 document. Following this requirement hierarchy enables the TMT engineering team to understand and visualize the interconnections between sub-systems, something that is essential when analyzing or changing requirements. This is enforced via the DOORS database by creating rules for linking requirements which are consistent with the document structure shown in figure 1 .

The DOORS literature describes several methods for creating links between requirements. These work well when the number of links to be created is small but become time consuming when a large number of links need to be created. To reduce this overhead we record traceability information as the requirements document is written (i.e. as a 'child' requirement is created, we record its 'parents'.) We then use a script written in the DOORS extension language (dxl) to generate the links automatically.

\subsection{Specific Metadata Recorded in TMT DOORS database}

In DOORS terminology a requirement is stored in DOORS as an object. Attached to each object are attributes that store the metadata for the requirement. Some of these are created automatically in categories specified by DOORS (these include the date the requirement was created, who created it, the date it was last changed) whilst some can be specified by the user. The following is a description of some of the user defined attributes used within the TMT project.

Sub-System Applicability - As explained in section 2, the TMT observatory is divided into 35 sub-systems. Each of the level 0 and level 1 requirements is assessed to determine whether a sub-system is either affected by the requirement or whether it contributes to accomplishing the requirement. This enables us to generate a list of all level 1 requirements applying to a specific sub-system and as the sub-system design requirements document is generated, we are able to check that each of the applicable level 1 requirements flows down adequately to that sub system. This is an essential step in checking that a sub-system DRD is complete.

Rationale - This is a description of why the requirement exists and an explanation of the justification for the requirement. When a requirement is changed, this attribute is also used to record the reason why it is changed. By recording the rationale we are able to improve the quality of the requirement, ensure that the intent of the requirement is clear and confirm that the requirement is necessary. The rationale also helps whenever a change is proposed to a requirement as it helps us remember why it exists in the first place.

Supporting Documentation - This attribute is included to document any analysis used to develop the requirement. By recording the inputs to a requirement we are able to confirm the validity of the requirement and as analysis is updated we can check for requirements that are affected.

Overall Verification Method - It is good practice to think about the verification method for a requirement as early as possible. We record the planned verification method alongside the requirement as the requirement is developed. This also assists us in the generation of verification documentation. DOORS allows the user to export specific objects and attributes to formats such as Microsoft excel; by recording the verification method as a DOORS attribute we can create a Verification Cross Reference Matrix directly from DOORS

Overall Verification Description - This attribute is used to capture details of the verification process envisaged for a particular requirement and is valuable for recording early ideas about possible verification processes. As with the overall verification method, this can be included in the VCRM generated from DOORS.

Overall Verification/Compliance Status - This attribute is included for inclusion in the VCRM to be generated from DOORS. 


\section{USING THE REQUIREMENT TRACEABILITY INFORMATION}

Requirement traceability is a combination of the links between different levels of requirement together with the requirement rationale and history. As the TMT design requirements have been developed and recorded in the DOORS database we have begun to use the traceability information to assess consistency of requirements, evaluate changes to requirements, examine verification methods, confirm interfaces are correct and check for completeness of requirement sets. The following section describes some of the ways we use or plan to use this information.

\subsection{Tracking Consistency between Level 0 and Level 1 Requirements}

As described in section 2, our level 1 documents respond to the level 0 science requirements and in some cases there may be known differences between them. DOORS uses the links between the SRD and ORD/OCD, to generate a report that identifies where any differences exist so that the engineering teams and Science Advisory Committee are aware of them. Having the links implemented in DOORS means that the relationship between level 0 and level 1 requirements is maintained even if either of the requirement sets is modified. The SRD requirements can be viewed alongside their level 1 'children' and changes quickly assessed for consistency. By using this information in combination with the TMT science flowdown matrix we have direct traceability between the level 1 requirements and the TMT science cases.

\section{$5.2 \quad$ Ensuring Completeness of Level 2 Requirements}

Each level 0 and level 1 requirement is assessed to establish which sub-systems it affects or which sub-systems are expected to contribute to its performance. As the sub-system requirement documents are developed, the applicability attribute is used to create a subset of OCD, ORD and OAD requirements that apply to the sub-system. These are used as a means of checking for completeness of the requirement set that is written for the sub-system. This flow down of requirements is checked periodically and will be assessed and reported at design reviews for each sub-system.

\subsection{Ensuring Completeness of Level 1 Requirements}

As the level 2 design requirement documents are generated, the links between them and the level 1 requirements are recorded in DOORS. By examination of the data in DOORS it becomes obvious wherever a level 2 requirement has no 'parent'. In some cases this may be appropriate, but it may also indicate that the level 1 document is deficient and that we need to define or modify a requirement in the OAD, ORD or OCD. It is important to address this situation to prevent the sub-system design from proceeding using incorrect assumptions. These 'orphan' requirements are tracked and reported at design reviews for each sub-system.

\subsection{Assessing Verification Processes Supporting Level 1 Verification}

By documenting the verification methods for sub-system requirements in DOORS it is possible to build up a picture of all the planned verification activities at level 2 and 3 that will support the verification of our level 1 requirements. The verification of level 1 performance may be supported by activities on several different sub-systems, but using the traceability information in DOORS to assess the total extent of verification assists the early planning of level 1 verification activities. It may indicate for example that sufficient verification tests are being done at sub-system level that system level verification can be limited to merely analyzing the results of the sub-system tests. It may also assist in guiding the way that verification activities are performed at sub-system level so that their benefit in demonstrating level 1 compliance is maximized.

\subsection{Changes to High Level Requirements}

TMT employs a change control process to manage the evolution of the level 1 documents. It is important to understand the effects of these changes on the sub-systems of the observatory before the changes are agreed. For every requirement change at level 1, DOORS is used to generate a report that identifies the sub-systems that could be affected by the change, the rationale for making the change and the level 2 requirements that are potentially affected. As updates to the level 2 DRDs aren't synchronized with the changes to level 1 documents we can use this report to track the level 2 requirements whose validity must be checked when the time comes to update the level 2 DRD.

\subsection{Monitoring for Non-compliances and Assessing effects on Observatory Performance}

TMT intends to continually monitor the status of compliance with level 0 and level 1 requirements and report this status at major system milestones. To maximize the benefits of this approach, sub-systems are expected to declare their 
compliance to requirements early in the sub-system development. This compliance status is a statement from the subsystem team or their subcontractor as to their expected compliance to a particular requirement. This compliance statement can be a simple yes or no against each individual DRD requirement. As this picture of compliance to level 2 requirements is created, the links to the $\mathrm{OAD}, \mathrm{ORD}$ and $\mathrm{OCD}$ will be used to develop an understanding of the level of compliance with the level 1 requirements and to identify areas where potential non-compliances or risk exist. It is hoped that this reporting will assist in mitigating the effects of non-compliances by adjusting requirements on other subsystems, for example by re-balancing system level budgets. By having links between the level 1 and level 0 requirements, the non-compliances at level 2 can be evaluated for their likely effect on meeting the science requirements.

\section{TMT BEST PRACTICES}

As the TMT requirements have evolved, it has been found necessary to develop some guidelines for the requirements to make sure that they are developed in a consistent manner. The following summarizes some of these guidelines.

\subsection{At what level should requirements reside}

The hierarchy of the level 1 documents described in section 2 is well understood and the definition of the scope of the document makes it clear in which of the level 1 documents a particular requirement should reside. The demarcation between level 1 and level 2 requirements is sometimes less clear so it is helpful to have some guidelines to ensure a consistent approach:

- If the requirement can only be satisfied by the contribution of one or more sub-systems it should not be included in a level 2 document.

- If the requirement describes a design implementation that potentially affects another sub-system or the system performance it should be included in the level 1 document

- Where a requirement can only be satisfied by the contribution of one or more sub-systems, the level 1 document should apportion that requirement between the involved sub-systems.

- A budget or performance allocation in the level 1 documents should ideally include a single term per subsystem. It should not attempt to apportion this term to the individual components that constitute the subsystem. This should be done at level 2 .

\subsection{Process Requirements versus Design Requirements}

As implied by their title, the level 1 and level 2 DRDs are intended to contain design requirements. These can be understood as requirements whose implementation is necessary to meet the performance goals of the observatory and whose accomplishment can be demonstrated via one of the agreed verification methods. This effectively excludes process requirements from the requirement flow down where their inclusion can deflect attention from the necessary technical requirements. Including unnecessary requirements including these process type requirements often leads to wasted effort as engineers have to spend time creating verification methods and recording their verification.

\subsection{Incomplete Requirements}

The TMT level 1 DRDs and several of the level 2 DRDs have by necessity been written before some of the operational, performance and design requirements could be fully defined. It is important however that wherever the need for a requirement is anticipated, a placeholder is included even if it is understood that the final requirement may be significantly different from the way this placeholder is defined.

\subsection{Requirements versus Interfaces}

Each ICD is recorded in DOORS and its elements stored as objects within the relevant DOORS module. Where a requirement is dependant on the correct accomplishment of the interface, links are generated between the ICD object and requirement object.

The contents of an ICD should be limited only to documenting the agreement of the implementation of the interface, where the manner of the implementation does not affect any other sub-system or the performance of the system. Level 2 requirements should not contain information that is under the control of another sub-system. If the information is critical 
to the accomplishment of the requirement then it should be included in the ICD where any changes to it must be agreed between the two sub-systems.

\subsection{Rules for linking requirements}

Whether or not links need to be created between two different requirements is not always clear and could be interpreted differently depending on the person creating the links. To try and maintain consistency in the way that requirements are linked. The following guidelines are used to establish whether or not two requirements should be linked.

- If a change to a higher level requirement could possibly affect the lower level requirement the two requirements should be linked. This may be quite an obscure link; for example the seismic conditions would be linked to our requirement for the minimum gaps between mirror segments

- If a lower level requirement contributes directly or indirectly to accomplishing a level 1 requirement then a link should be created

- If a level 2 requirement is derived from a combination of level 1 requirements then it should be linked to all those higher level requirements

- Our document hierarchy allows for level 2 requirements to be linked to requirements in any of the level 1 documents. This may lead to links being 'duplicated', i.e. the route from an ORD requirement to a level 2 requirement could be traced directly and also via link to the $\mathrm{OAD}$ and then on to the level 2 document

\section{EXAMPLE OF FLOWDOWN FROM SCIENCE CASE TO SUB-SYSTEM REQUIREMENTS}

The Science Flowdown for the Thirty Meter Telescope explains how the science cases are linked to the requirements of the SRD. As we have in place the links between the SRD and ORD and OCD, on to the OAD and then into the various level 2 and level 3 sub-system DRDs it is possible to examine how these science cases are influencing sub-system DRDs. The following example illustrates how rapidly the number of requirements increases as they are tracked from the highest level science requirement to sub-system component level.

The TMT science flowdown matrix identifies about forty SRD requirements that will contribute to supporting the follow up observation of Gama Ray Bursts. One of these is SRD requirement 0200:

'The telescope shall be able to move from any point in the sky to any other in less than 3 minutes and be ready to begin observing'

This requirement supports many other science cases identified in the TMT Detailed Science Case, contributes to the overall efficiency of the observatory and as shown in figure 3, affects many sub-systems of the observatory. Figure 3 uses data extracted from the DOORS database to illustrate how this flows down to the system and sub-systems. The requirement is defined in the SRD to allow the rapid movement of the telescope between science targets. This is an example of where a difference currently exists between the ORD and OCD and the SRD. This difference is well understood by the project science advisory committee and by the project engineering teams.

The SRD requirement can be traced directly to one requirement in the OCD and two in the ORD. The ORD breaks the SRD requirement into two separate requirements, the ability of the telescope and enclosure to move between two points anywhere on the sky within three minutes [REQ-1-ORD-1800] and the requirement for the entire system to move from one observation to another within 5 minutes [REQ-1-ORD-1805].

The first of these requirements is then derived into several velocity and acceleration requirements on enclosure and telescope moves in azimuth and elevation. One example of these, [REQ-1-OAD-1376] can then be traced to (amongst others) a requirement for the mount control system to provide a maximum azimuth rotation velocity. Another example of the flowdown of this requirement is to the primary mirror control system (M1CS). Here the level 2 DRD states a general performance requirement for M1CS components to operate over elevation velocities supporting telescope moves within three minutes. This in turn flows to a velocity requirement on the M1CS actuators that will ensure that any necessary repositioning of mirror segments occurs in a time consistent with overall telescope moves. 


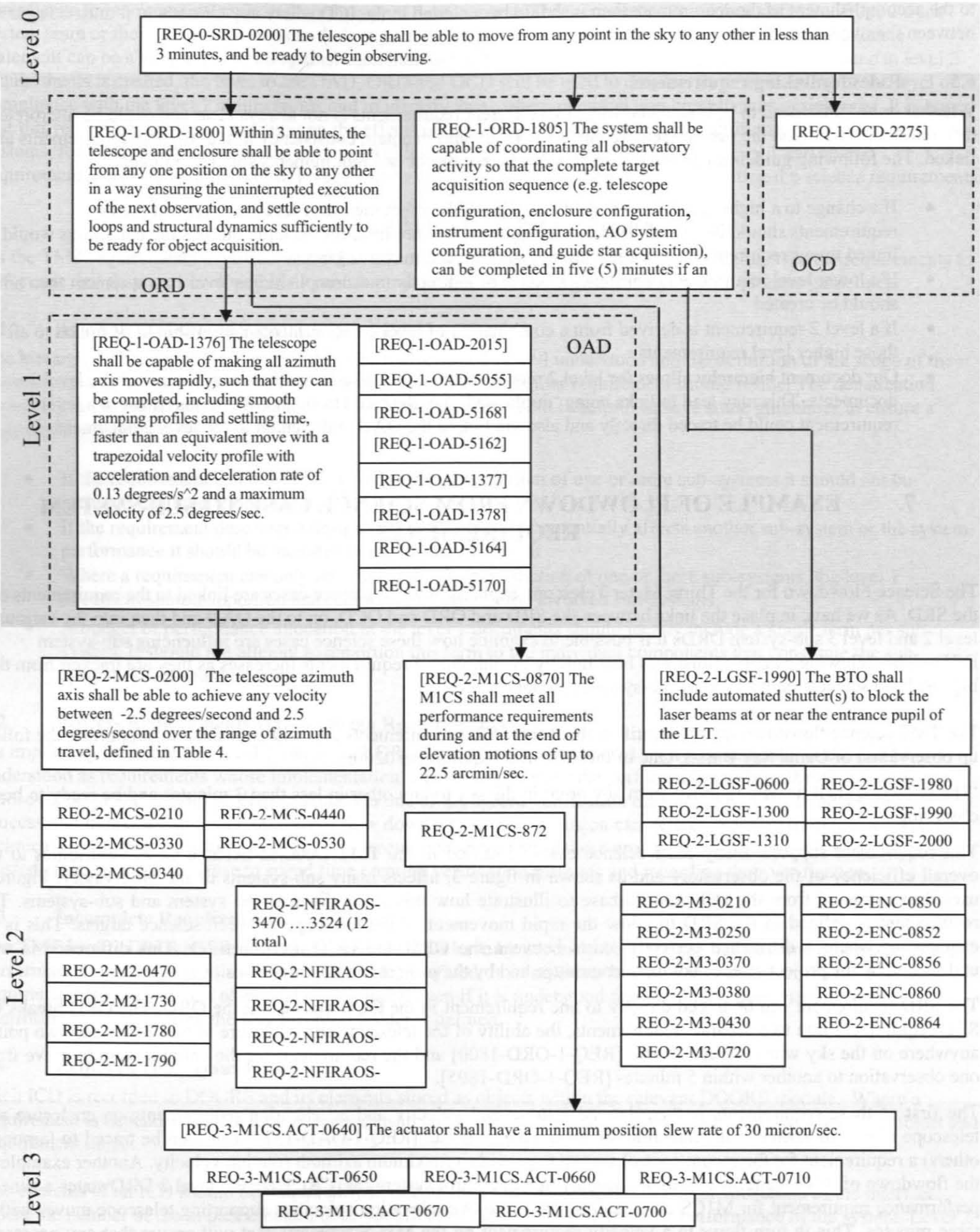

Figure 3 - Example of flowdown from Science Requirement to Sub-system (note: not all links shown) 
The second of the ORD requirements identified does not have any 'child' requirements in the OAD, but flows to multiple sub-system DRDs. The flow down to the Laser Guide Star Facility is used as an example. During telescope and enclosure slews between observations the lasers must be shuttered as the enclosure aperture motion is not synchronized with the telescope pointing. To allow observations to begin within the time specified by [REQ-1-ORD-1805], it is necessary to place this shutter 'downstream' of beam alignment and diagnostic equipment at the telescope top end. This allows the laser alignment to be maintained with the shutter closed and the laser to be propagated correctly immediately that the telescope and enclosure reach their desired positions and the shutter is opened.

In addition to these three examples, the SRD requirement can be traced to many other sub-systems' performance requirements. These include requirements on mirror figure surface settling time, laser pointing accuracy, AO component configuration times, and encoder read rates. It should be noted that as not all sub-system DRDs are available, the scope of the flow down of this requirement is expected to increase significantly once all requirements are defined.

\section{ACKNOWLEDGEMENTS}

The authors gratefully acknowledge the support of the TMT partner institutions. They are the Association of Canadian Universities for Research in Astronomy (ACURA), the California Institute of Technology and the University of California. This work was supported as well by the Gordon and Betty Moore Foundation, the Canada Foundation for Innovation, the Ontario Ministry of Research and Innovation, the National Research Council of Canada, the Natural Sciences and Engineering Research Council of Canada, the British Columbia Knowledge Development Fund, the Association of Universities for Research in Astronomy (AURA) and the U.S. National Science Foundation.

\section{REFERENCES}

[1] Angeli, G., "Systems engineering for the conceptual design of the Thirty Meter Telescope" in [Modeling, Systems Engineering and Project Management for Astronomy II], Proc SPIE 6271-10F (2006)

[2] Angeli, G., Roberts, S. and Vogiatzis, K., "Systems Engineering for the Preliminary Design of the Thirty Meter Telescope" in [Modeling, Systems Engineering and Project Management for Astronomy III], Proc SPIE 7017-02 (2008)

[3] TMT Science-based Requirements Document (SRD, www.tmt.org/sites/default/files/SRD-CCR18-Science-BasedRequirements-Document.pdf)

[4] TMT Operations Concept Document (OCD, www.tmt.org/sites/default/files/documents/application/pdf/ocdccr08.pdf)

[5] TMT Observatory Requirements Document (ORD, www.tmt.org/sites/default/files/documents/application/pdf/ordccr22.pdf.pdf)

[6] TMT Observatory Architecture Document (OAD, www.tmt.org/sites/default/files/documents/application/pdf/oadccr21.pdf.pdf)

[7] Simard, L. and Crampton, D., "Science Flowdown for the Thirty Meter Telescope," in [Ground-based and Airborne Instrumentation for Astronomy III], McLean, I. S., Ramsay, S. K., and Takami, H., eds., Proc. SPIE 7735-213 (2010).

[8] TMT Detailed Science Case, (DSC, http://www.tmt.org/sites/default/files/TMT-DSC-2007-R1.pdf) 